\title{
VORWORT DES VERFASSERS
}

Die Informationstheorie im weiteren Sinne und die speziell im vorliegenden Buch abgehandelte statistische Theorie der elektrischen Nachrichtenübertragung als Informationstheorie im engeren Sinne sind Teilgebiete einer sich neu herausbildenden wissenschaftlichen Disziplin, der Kybernetik. Die jeder Wissenschaft eigene Abstraktion und Zusammenfassung äußerlich verschiedener Erscheinungen in einer gemeinsamen Theorie ist im Falle der Informationstheorie besonders ausgeprägt. Der Verfasser hofft, durch die gewählte Art der Darstellung, ergänzt durch zahlreiche Beispiele, diese Schwierigkeit für das Verstehen und Anwenden der Theorie nach Möglichkeit herabgemindert zu haben.

Für die. Unterstützung bei diesem Bemühen dankt der Verfasser besonders Herrn Professor Dr.-Ing. H. Frühauf und Herrn Professor Dr. Kämmerer. Dank gebührt weiter den Herren Prof. Dr. Lanoe und Dr. Wunsch für wertvolle Hinweise und Diskussionen sowie dem Verlag für die gute Gestaltung des Buches.

Berlin, im Juni 1963 